\title{
A BIOCHEMICAL STUDY OF THE EFFECT OF HIGH MOLECULAR WEIGHT HYALURONIC ACID IN MANAGEMENT OF TEMPOROMANDIBULAR JOINT OSTEOARTHRITIS IN RATS
}

\author{
Yasmine M. Tolba ${ }^{1 *} B D S$, Samia S. $\operatorname{Omar}^{2} P h D$, Dina A. Nagy ${ }^{3} P h D$
}

\begin{abstract}
INTRODUCTION: Osteoarthritis is one of the most common degenerative diseases affecting the joints. Adjuvant- induced arthritis (AIA) is an experimental model of polyarthritis which has been widely used for preclinical testing of several anti-arthritic agents and investigating the pathogenesis of arthritis. A modified model of AIA was used in this study, where the disease was induced in one joint. Hyaluronic acid deals with the degenerative effects of arthritis from 3 different aspects: mechanically by maintaining lubrication, metabolically by providing nutritional materials to the articular disc and biologically by decreasing inflammatory effects.

OBJECTIVES: to study the biochemical actions of high molecular weight hyaluronic acid (HMWHA) in management of temporomandibular joint (TMJ) adjuvant induced arthritis by detecting matrix metalloproteinase-3 (MMP3) concentration in TMJ tissue extract.

MATERIALS AND METHODS: 14 male albino rats were divided into 2 equal groups and in each group a split mouth design was used.

- Study I: Arthritis was induced in 7 animals through a single intra-articular injection of Complete Freund's Adjuvant (CFA) in the left TMJs. (50pl)

-Study II: Arthritis was induced in the other 7 animals through a single intra-articular injection of CFA and treated with an intra-articular injection of HMWHA into their left TMJs, once a week for total of three injections.

7 animals were sacrificed on day 7 of the disease while the other 7 animals were sacrificed one week after the last injection of HMWHA.

Elisa test was performed to detect MMP 3 which is considered as a biomarker of the disease progression

RESULTS: The concentration of MMP-3 of study I (diseased group) on day 7 was found to be significantly higher than that of study II (treated group) on day 28.

CONCLUSIONS: High molecular weight hyaluronic acid is capable of management of osteoarthritic TMJs.

KEYWORDS: TMJ, Arthritis, High molecular weight hyaluronic acid, MMP-3.
\end{abstract}

1- Demonstrator of Oral Biology, Faculty of Dentistry, Alexandria University.

2- Professor of Oral Biology, Faculty of Dentistry, Alexandria University.

3- Lecturer of Oral Biology, Faculty of Dentistry, Alexandria University.

*Corresponding author:

E-mail: yasminetolba@hotmail.com

\section{INTRODUCTION}

Osteoarthritis (OA) is one of the most common degenerative diseases affecting the temporomandibular joint (TMJ) (1). TMJ osteoarthritis (TMJ-OA) affects approximately $15 \%$ of the world's population. OA occurs more often in elderly patients; however, it can affect patients at any age (2). The main symptom of an arthritic joint is dull pain. The inflammatory clinical features of the disease also include: masticatory muscles and TMJ tenderness, joint instability, stiffness, joint sounds and decreased range of joint motion $(3,4)$.

Excessive mechanical stress and decreased adaptive capacity are among the main causes of the degenerative events occurring in the TMJ. As a consequence, to functional overloading, hypoxia occurs, breaking down of hyaluronic acid present in the synovial fluid and vascular endothelial growth factor (VEGF) is secreted (3).

In the case of OA, high levels of inflammatory mediators have been detected in the synovial fluid(SF) (2). Inflammatory mediators such as interleukin-1b (IL-1b) and tumor necrosis factor alpha (TNF- $\alpha$ ) are released. These cytokines are able to cause the destruction of cartilage and decreasing the concentration and molecular weight of HA. This affects the viscosity and nature of SF negatively (5). They are also linked to the activation of matrix metalloproteinases (MMPs) (6).
Matrix metalloproteinases were divided into six groups according to their function and target substrate: collagenases (MMP-1, 8, -13), gelatinases (MMP-2 and -9), stromelysins (MMP-3, -10, -11), matrilysins (MMP-7, -26), membrane-type MMPs (MT-MMPs), and other nonclassified MMPs. MMPs are expressed in very low levels in normal joints; however, they are mainly secreted by arthritic joints (7).

Matrix metalloproteinase-3 (MMP-3) is secreted by both chondrocytes and synovial cells. It does not only degrade the extracellular matrix of cartilage, but also activates other MMPs (8). MMP-3 shares in the activation of proMMP-1, thus giving MMP-3 a dual role in matrix destruction by affecting both collagen and proteoglycans (9).

Hyaluronic acid (HA) is an anionic disaccharide repeats of N-acetyl glucosamine and glucuronic acid. It belongs to the glycosaminoglycans (GAGs), but it is the only nonsulfated GAG. It contains up to about 25,000 repeating disaccharide units. HA has normally a high molecular weight that can reach up to $107 \mathrm{Da}$. (5)

Hyaluronic acid is a natural component of the synovial fluid and the extracellular matrix of cartilage (10). It is also considered one of the most hydrophilic molecules in nature (11). The mechanism of action of HA may not yet be clearly understood, however, it possesses multiple protective functions in the cartilage. In this study, we examine the level 
of MMP-3 concentration in osteoarthritic TMJs before and after treatment using HMWHA. However, the null hypothesis of this study proposes that no effect will be obtained by using high molecular weight hyaluronic acid on the changes induced by arthritis in rat model.

\section{MATERIALS AND METHODS}

The Ethical Committee of the Faculty of Dentistry Alexandria University approved the protocol of this research. 14 adult male albino rats weighting about 300-400 grams were used in this study. These animals were obtained from the Medical Research Institute, Alexandria University. Animals were housed in specially designed wire mesh bottom cages and were supplied a regular diet throughout the duration of the experiment.

\section{Induction of osteoarthritis:}

Induction of arthritis was carried out in the left TMJs of all animals by a single intra-articular injection of $50 \mu \mathrm{l}$ (12) of Complete Freund's Adjuvant (CFA), diluted 1:1(oil: saline) (13) (14) (Fig.1) CFA used was of a concentration of $1 \mathrm{mg} / \mathrm{ml}$ heat killed mycobacterium tuberculosis. The day of the induction was considered 0 day.

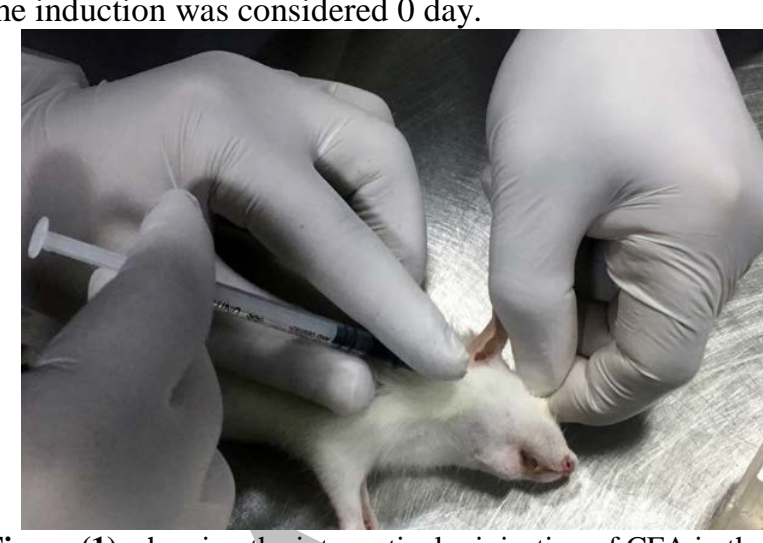

Figure (1): showing the intra-articular injection of CFA in the TMJ.

The animals were divided into 2 equal groups and in each group a split mouth design was used.

- Study I: consisted of 7 left TMJs with induced arthritis.

- Study II: consisted of 7 left TMJs with induced arthritis that were treated with a $50 \mu \mathrm{l}$ intra-articular injection of HMWHA (Optivisc, London, UK) (2.3 to 2.5 million daltons), once a week for total of three injections on days 7 , 14 and $21(15,16)$. HA was provided in the form of pre-filled sterile syringes. Each syringe was intended for a single use only. The calculated volume was withdrawn for the intraarticular injections immediately after opening the syringe and any excess was discarded.

Treated animals received $300 \mathrm{mg} / \mathrm{kg}$ Acetaminophen (Panadol, Leeds, UK) orally (17) in drinking water, after intra-articular CFA injection to prevent painful symptoms. The animals were anesthetized during the induction of the arthritis and HMWHA injections by inhalational anesthesia (Di ethyl ether). Animals were sacrificed using an overdose of inhalation anesthesia to obtain tissue samples needed for ELISA testing before and after the treatment detection of MMP-3.

\section{Biochemical analysis:}

Elisa test (Glory Science Co, Texas, USA) was performed at Medical Technology Center for Research and Services, Medical Research Institute, Alexandria University to determine presence of MMP 3, which is considered as a biomarker of the disease progression.

Tissue samples were obtained from both study I and II. First on day 7 before the first HMWHA injection, 7 animals were sacrificed. Secondly on the day 28, 7 other animals were sacrificed as well. Tissue extracts were rinsed with Phosphate buffer solution (PBS) to remove excess blood and then samples were cut into $1-2 \mathrm{~mm}$ pieces in size. Samples were grinded using a tissue homogenizer machine. (Fig.2) Tissue extract was centrifuged for 20 minutes at speed 2000-3000 rpm. Finally, the supernatant is extracted to be used. (18) The kit used was a double-antibody sandwich enzyme-linked immunosorbent assay (ELISA) and MMP-3 levels were measured according to manufacturer's instructions. (Fig. 2 and 3)

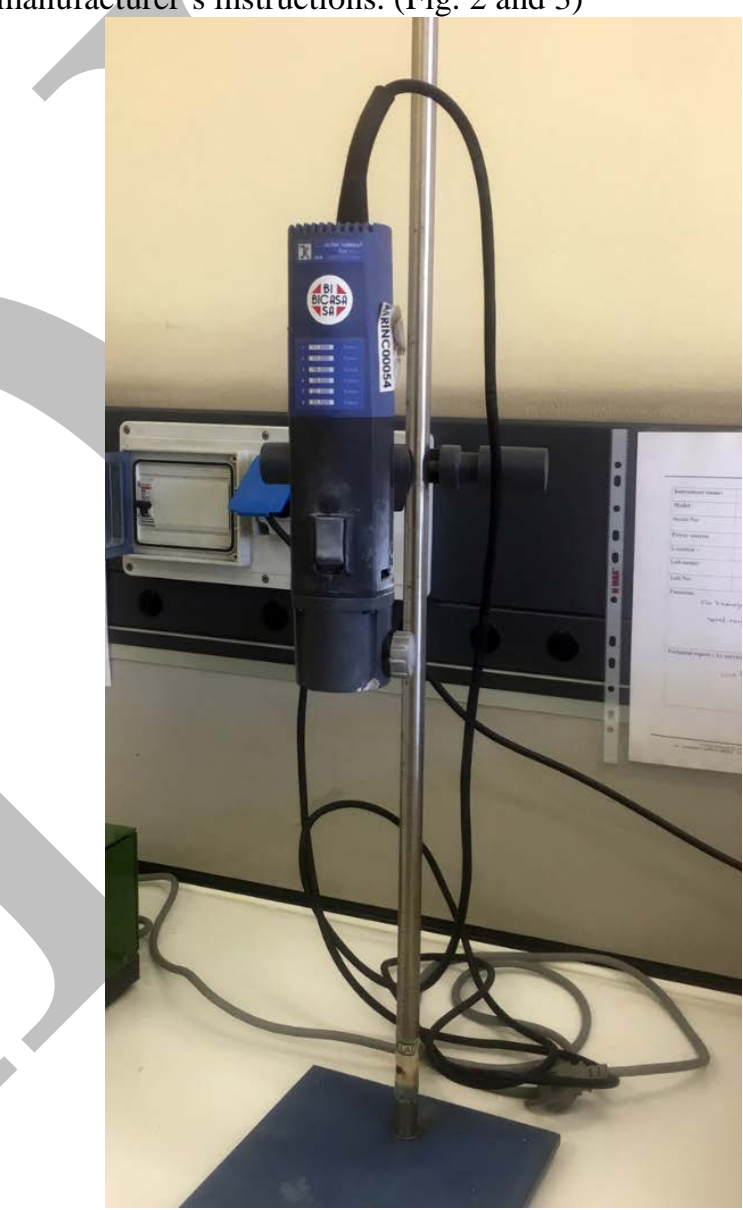

Figure (2): showing the tissue homogenizer machine used to grind the samples.

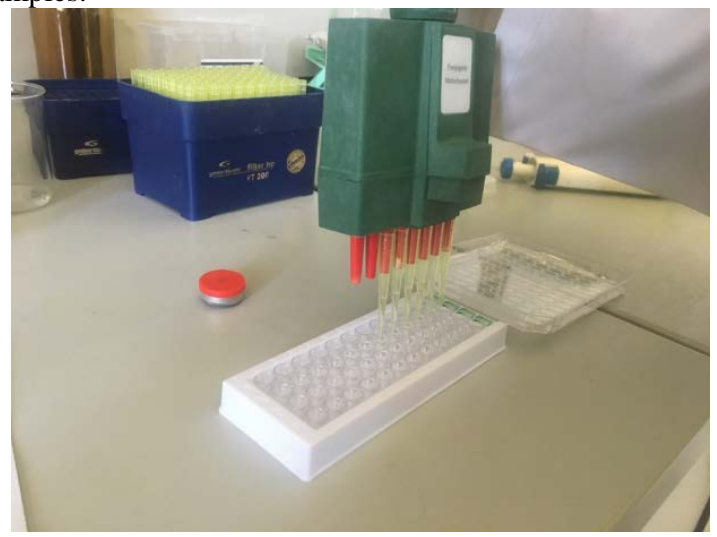

Figure (3): showing fixed volume Finnpipette used during the ELISA procedure. 


\section{STATISTICAL ANALYSIS:}

Descriptive statistics were calculated including mean and standard deviation (SD). The difference between the concentrations of MMP3 on day 7 of the diseased group and on day 28 of the treated group was measured using unpaired t- test. A (P) value less than 0.05 was considered significant.

\section{RESULTS}

The results of the concentrations of MMP-3 in both groups (study I and II) are presented in (Table 1).

The first readings show the concentrations in $\mathrm{ng} / \mathrm{ml}$ of MMP-3 on day 7 in the diseased group (study I). The mean concentration of MMP-3 in study I is (0.227) and standard deviation (SD) (0.0157). The second readings show the concentrations in ng/ml of MMP-3 on day 28 in the treated group after 3 injections of HMWHA (study II). The mean concentration in the treated group is $(0.208)$ and SD is (0.0126).

The difference between the concentrations of MMP-3 in study I (Disease group) on day 7 and treated study II (Treated group) on day 28 was calculated using unpaired $t$ test. $(\mathrm{P}=0.03)$ There was a significant difference between the MMP-3 concentration on day 7 and day 28. $(\mathrm{P}<0.05)$ (Fig. 4)

Table (1): showing the concentrations of MMP-3 in both groups.

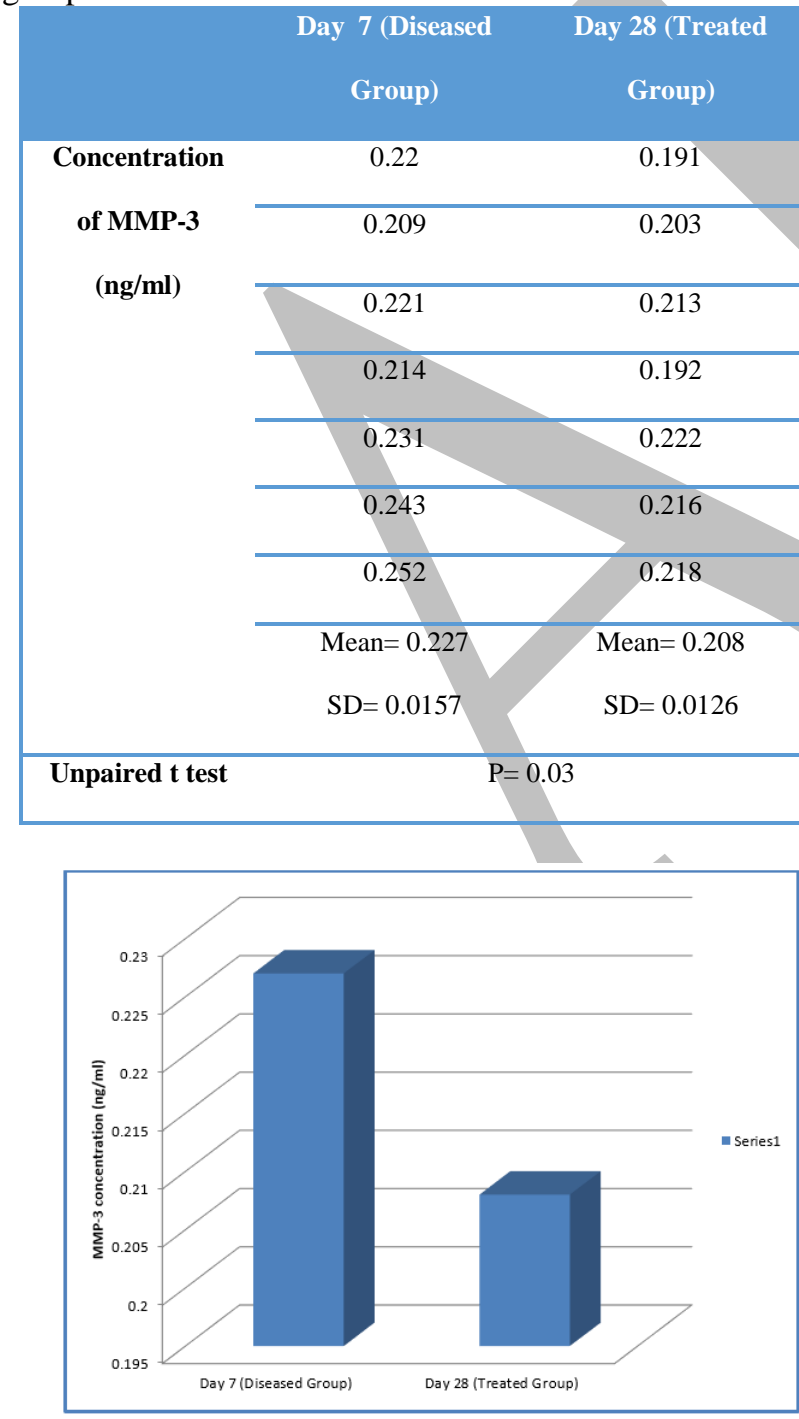

Figure (4): Bar representation of the values of MMP-3 concentration in the diseased and treated groups.

\section{DISCUSSION}

Osteoarthritis is an inflammatory degenerative disease affecting the joints. An imbalance between the anabolic and catabolic activities leads to destruction of the cartilage and bone of the TMJ.

The most common symptom of an arthritic joint is dull pain, which may be caused due to the surrounding muscles contraction as a reflex action to intra-articular injury or pathology, thus protecting it from further damage. Pain also could arise due to the bone destruction occurring (3).

Vascular endothelial growth factor (VEGF) is released by chondrocytes of the mature and hypertrophic cell layers of the condyle due to prolonged mechanical loading. This leads to increasing the vascularity in the synovium and vascular invasion of the joint (19). The expression of VEGF by osteoblasts in OA might explain the enhanced osteoblastic activity and subsequently subchondral bone sclerosis and osteophytes formation (20). VEGF is also capable of inducing osteoclasts and acts in cartilage as an autocrine inducer of MMPs (6).

This study was conducted to examine the concentration of MMP-3 in TMJ tissue extracts of rats after arthritis induction and after treatment using 3 injections of HMWHA. Arthritis was induced using a single injection of CFA in the left TMJs. Local administration was suggested to guarantee the occurrence of monoarthritis and avoid unnecessary adverse effects on the animal systemically. Monoarthritis model was tested by both Butler et al. (21) and Donaldson et al. (22) in their studies to develop an adjuvant- induced arthritis model in tibio-tarsal joint of rats. The ELISA assessment showed a significant increase in MMP-3 concentration on day 7 of disease when compared with samples from treated group.

High molecular weight HA has chondroprotective effects and decreased the progression of cartilage and bone degeneration. HMWHA stimulates chondrocyte proliferation, production of cartilage matrix, and inhibition of protease. Intraarticular injection of HMWHA offers nutrition, lubrication and reduces mechanical stress of the TMJ. This is thought to be supported by Campo et al. (23) and Machado et al. (24)

In agreement with the current results, Hashizume et al. (1) found that the production of MMP-3 was significantly suppressed by HA treatment in their study. However, they examined the synovial fluid of rabbit OA model. In addition, the results obtained in this study are in accordance with the findings of, Chen et al. (8) The immunohistochemical analysis performed by these authors showed that synovium of osteoarthritic knees showed significantly higher MMP-3 expression when compared with control group. Its expression was also positively correlated with the severity of OA.

Moreover, in accordance with the findings of our study, Triantaffilidou et al. (25) conducted an experiment on 25 patients suffering from TMJ-OA. The patients underwent a cycle of 5 intra-articular injections of HA. The results were found satisfactory after 1-year follow-up. On the other hand, Jørgensen et al. (26) performed in 2014 a randomized, placebo-controlled, double-blind study of 337 patients diagnosed with one knee osteoarthritis. Patients received 5 injections of saline or HA based on the group they were assigned to. After 1 year follow up, no significant difference was found between intra-articular hyaluronan and placebo treatment. The difference between the previous results and 
the current findings may be due to the difference in the experimental model, since they used human subjects while the current study was conducted on rats.

The mechanism of action of HA may not yet be clearly understood, however, it possesses multiple protective functions in the cartilage. Providing further confirmation to the positive action of HMWHA, studies by Duygu et al. (15) and Lemos et al.(16)showed that high molecular weight HA had chondroprotective effects and decreased the progression of cartilage and bone degeneration.

\section{CONCLUSION}

The intra-articular injections of HMWHA showed promising results in the osteoarthritic TMJ of rat model.

\section{CONFLICT OF INTEREST}

The authors declare that they have no conflicts of interest.

\section{REFERENCES}

1. Hashizume M, Koike N, Yoshida H. High molecular weight hyaluronic acid relieved joint pain and prevented the progression of cartilage degeneration in a rabbit osteoarthritis model after onset of arthritis. Mod Rheumatol. 2010;20:432-8.

2. Kalladka M, Quek S, Heir G, Eliav E, Mupparapu M, Viswanath A. Temporomandibular joint osteoarthritis: Diagnosis and long-term Conservative management: A topic review. J Indian Prosthodont Soc. 2014;14:6-15.

3. Tanaka E, Detamore MS, Mercuri LG. Degenerative Disorders of the Temporomandibular Joint: Etiology, Diagnosis, and Treatment. J Dent Res. 2008;87:296-307.

4. Bansal M. Prevalence and diagnostic features of osteoarthrosis of the temporomandibular joint: a review. Int J Res Orthop. 2016;2:1-4.

5. Iturriaga V, Vásquez B, Manterola C, del Sol M. Role of Hyaluronic Acid in the Homeostasis and Therapeutics of Temporomandibular Joint Osteoarthritis. Int J Morphol. 2017;35:870-6.

6. Tanne K, Okamoto Y, Su S-C, Mitsuyoshi T, AsakawaTanne Y, Tanimoto K. Current status of temporomandibular joint disorders and the therapeutic system derived from a series of biomechanical, histological, and biochemical studies. APOS Trends Orthod. 2015;5:4-21.

7. Jabłońska-trypuć A, Matejczyk M, Rosochacki S. Matrix metalloproteinases (MMPs), the main extracellular matrix (ECM) enzymes in collagen degradation, as a target for anticancer drugs enzymes in collagen degradation, as a target for anticancer drugs. J Enzyme Inhib Med Chem. 2016;31:177-83.

8. Chen J, Huang J, Du W, Tong P. Expression and significance of MMP3 in synovium of knee joint at different stage in osteoarthritis patients. Asian Pac J Trop Med. 2014;7:297-300.

9. Burrage PS, Mix KS, Brinckerhoff CE. Matrix metalloproteinases: role in arthritis. Front Biosci. 2006;11(July 2016):529-43.

10. Kogan G, Šoltés L, Stern R, Gemeiner P. Hyaluronic acid: A natural biopolymer with a broad range of biomedical and industrial applications. Biotechnol Lett. 2007;29:17-25.

11. Necas J, Bartosikova L, Brauner P, Kolar J. Hyaluronic acid (hyaluronan): A review. Vet Med (Praha). 2008;53:397411.
12. Harper RP, Kerins CA, McIntosh JE, Spears R, Bellinger LL. Modulation of the inflammatory response in the rat TMJ with increasing doses of complete Freund's adjuvant. Osteoarthr Cartil. 2001;9:619-24.

13. Kuroki Y, Honda K, Kijima N, Wada T, Arai Y, Matsumoto $\mathrm{N}$, et al. In vivo morphometric analysis of inflammatory condylar changes in rat temporomandibular joint. Oral Dis. 2011;17:499-507.

14. Lemos GA, Rissi R, de Souza Pires IL, de Oliveira LP, de Aro AA, Pimentel ER, et al. Low-level laser therapy stimulates tissue repair and reduces the extracellular matrix degradation in rats with induced arthritis in the temporomandibular joint. Lasers Med Sci. 2016;31:1051-9.

15. G.Duygu, N.Güler, B.Çam, M.Kürkçü. The effects of high molecular weight hyaluronic acid ( Hylan G-F 20 ) on experimentally induced temporomandibular joint osteoartrosis: part II. Int J Oral Maxillofac Surg. 2011;40:1406-13.

16. Lemos GA, Rissi R, Pimentel ER, Palomari ET. Effects of high molecular weight hyaluronic acid on induced arthritis of the temporomandibular joint in rats. Acta Histochem. 2015;117:566-75.

17. Considerations A, Specific S, Formulary V, Introduction I. Veterinary Anesthetic and Analgesic Formulary. 2012;1-22.

18. Yang C, Lin C, Wang H, Lyu S. Matrix Metalloproteases and Tissue Inhibitors of Metalloproteinases in Medial Plica and Pannus-like Tissue Contribute to Knee Osteoarthritis Progression. PLoS One. 2013;8:1-11.

19. Kuyinu EL, Narayanan G, Nair LS, Laurencin CT. Animal models of osteoarthritis: classification, update, and measurement of outcomes. J Orthop Surg Res. 2016;1-27.

20. Corrado A, Neve A, Cantatore FP. Expression of vascular endothelial growth factor in normal, osteoarthritic and osteoporotic osteoblasts. Clin Exp Med. 2013;13:81-4.

21. Butler SH, Godefroy F, Besson JM, Weil-Fugazza J. A limited arthritic model for chronic pain studies in the rat. Pain. 1992;48:73-81.

22.Donaldson LF, Seckl JR, McQueen DS. A discrete adjuvant-induced monoarthritis in the rat: effects of adjuvant dose. J Neurosci Methods. 1993;49:5-10.

23. Campo GM, Avenoso A, Nastasi G, Micali A, Prestipino V, Vaccaro $\mathrm{M}$, et al. Hyaluronan reduces in $\mathrm{fl}$ ammation in experimental arthritis by modulating TLR-2 and TLR-4 cartilage expression. Biochim Biophys Acta. 2011;1812:1170-81.

24. Machado E, Bonotto D, Cunali PA. Intra-articular injections with corticosteroids and sodium hyaluronate for treating temporomandibular joint disorders: a systematic review. Dental Press J Orthod. 2013;18:128-33.

25. Triantaffilidou K, Venetis G, Bika O. Efficacy of hyaluronic acid injections in patients with osteoarthritis of the temporomandibular joint. A comparative study. J Craniofac Surg. 2013;24:2006-9.

26. Jørgensen A, Stengaard-pedersen K, Simonsen O, Eriksen C, Bliddal H, Pedersen NW, et al. Intra-articular hyaluronan is without clinical effect in knee osteoarthritis: a multicentre, randomised, placebo-controlled, double-blind study of 337 patients followed for 1 year. 2010;69:1097102. 